\title{
Profissionais de enfermagem de nível médio: série temporal salarial em dez anos
}

\author{
Licensed practical nurses professionals: ten year salary time series \\ Profesionales de enfermería de nivel medio: series temporales de sueldos de diez años
}

Recebido: 24/11/2021 | Revisado: 02/12/2021 | Aceito: 03/12/2021 | Publicado: 13/12/2021

Edivane Pedrolo

ORCID: https://orcid.org/0000-0003-2467-9516 Instituto Federal de Educação, Ciência e Tecnologia do Paraná, Brasil E-mail: edivane.pedrolo@ifpr.edu.br

Tangriane Hainiski Ramos

ORCID: https://orcid.org/0000-0002-6641-9715 Instituto Federal de Educação, Ciência e Tecnologia do Paraná, Brasil E-mail: tangriane.ramos@ifpr.edu.br

Nadine de Biagi Ziesemer

ORCID: https://orcid.org/0000-0002-5001-2347 Instituto Federal de Educação, Ciência e Tecnologia do Paraná, Brasil E-mail: nadine.biagi@ifpr.edu.br

Radamés Boostel

ORCID: https://orcid.org/0000-0003-2678-0001 Instituto Federal de Educação, Ciência e Tecnologia do Paraná, Brasil E-mail: radames.boostel@ifpr.edu.br

Rafael Haeffner

ORCID: https://orcid.org/0000-0001-8848-6460 Instituto Federal de Educação, Ciência e Tecnologia do Paraná, Brasil E-mail: Rafael.haeffner@ifpr.edu.br

\begin{abstract}
Resumo
A enfermagem brasileira vem lutando por reconhecimento profissional, carga horária mais humana e política salarial mais justa. Neste contexto estão inseridos também os profissionais de enfermagem de nível médio. Objetivou-se analisar a série temporal salarial dos auxiliares e técnicos em enfermagem em seu primeiro emprego, no período de 2010 a 2019. Realizou-se estudo epidemiológico retrospectivo com egressos de um curso técnico em enfermagem da rede federal de educação dos anos de 2010 a 2019. Foram incluídos 143 egressos, sendo a maioria mulheres jovens. A remuneração média foi de $\mathrm{R} \$ 1.702,92$ (mínimo de $\mathrm{R} \$ 790,00$ e máximo de $\mathrm{R} \$ 4.000,00$ ). A série temporal salarial demonstrou tendência estacionária no período analisado, enquanto o salário-mínimo teve tendência crescente. A remuneração dos técnicos/auxiliares em enfermagem encontra-se aquém do que anseia a categoria profissional. A existência de um projeto de lei que determina o piso salarial da categoria e a jornada de trabalho vem ao encontro com a valorização destes trabalhadores; e sua implementação como política pública tende a fortalecer este coletivo. A estagnação salarial evidenciada nas séries temporais pode representar um risco de evasão profissional para outras modalidades de trabalho.
\end{abstract}

Palavras-chave: Recursos humanos de enfermagem; Técnicos de enfermagem; Salários e benefícios; Remuneração; Mercado de trabalho.

\begin{abstract}
The Brazilian nursery has been fighting for professional recognition, a more humane workload and a fairer salary policy. In this context, mid-level nursing professionals are also included. The objective of this study was to analyze a time series of salaries of nursing assistants and technicians in their first job, from 2010 to 2019. A retrospective epidemiological study was carried out with graduates of a nursing technician course from the federal education network from 2010 to 2019. The 143 graduates were included, most of them young women. The average remuneration was $R \$ 1,702.92$ (minimum of $R \$ 790.00$ and maximum of $R \$ 4,000.00$ ). The wage time series is a constant trend in the period analyzed, while the minimum wage has an increasing trend. The remuneration of nursing technicians/auxiliaries is below what the professional category wants. The existence of a bill that determines the category's wage floor and a working day is in line with the valuation of working workers; and its implementation as a public policy tends to strengthen this collective. The salary stagnation evidenced in the time series may represent a risk of professional evasion for other types of work.
\end{abstract}

Keywords: Nursing staff; Licensed practical nurses; Salaries and fringe benefits; Remuneration; Job market. 


\begin{abstract}
Resumen
La enfermería brasileña ha luchado por el reconocimiento profesional, una carga de trabajo más humana y una política salarial más justa. En este contexto, también se incluyen los profesionales de enfermería de nivel medio. El objetivo fue analizar la serie temporal de sueldos de los auxiliares y técnicos de enfermería en su primer empleo, de 2010 a 2019. Se realizó un estudio epidemiológico retrospectivo con egresados de un curso de técnico en enfermería de la red educativa federal de 2010 a 2019. 143 se incluyeron graduados, la mayoría mujeres jóvenes. La remuneración promedio fue de $\mathrm{R} \$ 1.702,92$ (mínimo de $\mathrm{R} \$ 790,00$ y máximo de $\mathrm{R} \$ 4.000,00$ ). La serie temporal de salarios mostró una tendencia estacionaria en el período analizado, mientras que el salario mínimo mostró una tendencia creciente. La retribución de los técnicos / auxiliares de enfermería está por debajo de lo que desea la categoría profesional. La existencia de un proyecto de ley que determine el piso salarial de la categoría y la jornada laboral está en consonancia con la apreciación de estos trabajadores; y su implementación como política pública tiende a fortalecer este colectivo. El estancamiento salarial evidenciado en la serie temporal puede representar un riesgo de evasión profesional hacia otros tipos de trabajo.

Palabras clave: Recursos humanos de enfermería; Técnicos de enfermería; Salarios y beneficios; Remuneración; Mercado de trabajo.
\end{abstract}

\title{
1. Introdução
}

O reconhecimento da enfermagem enquanto profissão essencial na atenção à saúde tem sido foco de iniciativas nos últimos anos. A mais emblemática delas, lançada em 2018, foi intitulada Campanha Nursing Now. Promovida pela Organização Mundial da Saúde (OMS), pelo Conselho Internacional de Enfermagem e pela UK All Party Parliamentary Group on Global Health, do Reino Unido, tem como metas o incentivo à formação e a valorização da enfermagem enquanto profissão (Cassiani \& Lira, 2018). De forma complementar, o ano de 2020 foi proclamado pela OMS como o “Ano da Enfermagem" (Silva, 2019).

No Brasil, a Campanha Nursing Now foi propagada pelo Conselho Federal de Enfermagem (Cofen), em parceria com o Centro Colaborador da OPAS/OMS para o Desenvolvimento da Pesquisa em Enfermagem. Além das metas globais, no Brasil esta iniciativa teve como foco a luta por reconhecimento profissional, mas em especial por melhores condições de trabalho, por uma carga horária mais humana e por uma política salarial mais justa (Silva, 2019), sendo estas lutas históricas da enfermagem desde que se consolidou como profissão.

O profissional de enfermagem representa $50 \%$ do contingente de profissionais no setor saúde no Brasil (Machado et al, 2015), portanto a força de trabalho dos auxiliares, técnico e enfermeiros, sem dúvidas, alavancam o atendimento à saúde em seus diferentes níveis de atenção, bem como, determinam o alcance de metas nos âmbitos governamentais e/ou privados.

Seguramente, esta classe profissional possui uma vasta área de atuação, o que expande as possibilidades de empregabilidade, sejam elas nos âmbitos da atenção primária, secundária ou terciária, nos diferentes ciclos de vida do ser humano; e esta atividade profissional compreende desde ações educativas em saúde, relacionadas à promoção e melhoria de qualidade de vida, até o cuidado de alta complexidade, que envolve a atenção do ser humano com risco de morte e a manipulação de equipamentos de alta complexidade (Anderson \& Schneider, 2016).

A rede suplementar de saúde possui uma ampla estrutura de atendimento e, juntamente com o Sistema Único de Saúde (SUS), representa um importante local de inserção dos profissionais de enfermagem no mercado de trabalho. Por ser regido, em sua maioria, pela Consolidação das Leis do Trabalho (CLT), a contratação de profissionais ocorre de forma mais rápida e menos burocrática, quando comparada ao serviço público, que exige a realização de concursos. Pesquisa que buscou contrastar organizações públicas e privadas brasileiras observou o acesso à tecnologia e a remuneração como atrativos para o ingresso na rede privada, enquanto a estabilidade destaca-se como um diferencial no serviço público (Giordano, 2020).

Estudo que analisou a tendência temporal de recursos humanos para o SUS e para Saúde Suplementar no Brasil, no período de 2005 a 2016, demonstrou que houve tendência crescente do quantitativo de profissionais da saúde de todas as categorias analisadas na saúde suplementar. Quando analisados somente os dados do Paraná, houve crescimento na demanda 
Research, Society and Development, v. 10, n. 16, e346101623840, 2021

por técnicos em enfermagem tanto no setor público quanto privado, contudo neste a demanda foi maior (Campony et al, 2020).

Considerando que a saúde suplementar engloba instituições com fins lucrativos, e que a equipe de enfermagem compreende metade do quantitativo de profissionais em uma instituição de saúde, ${ }^{1}$ seja ela pública ou privada, indaga-se sobre a remuneração paga a esta categoria de profissionais pelo exercício de sua profissão. Assim, o objetivo foi analisar a série temporal salarial dos auxiliares e técnicos em enfermagem em seu primeiro emprego, no período de 2010 a 2019.

\section{Metodologia}

Realizou-se um estudo epidemiológico retrospectivo. A população investigada compreendeu egressos de um curso técnico em enfermagem ofertado por uma instituição federal na cidade de Curitiba-PR, entre os anos de 2010 e 2019, que compreendem as primeiras dez turmas formadas pela referida instituição.

Foram considerados como critério para inclusão dos participantes: ter concluído o curso técnico em enfermagem na instituição pesquisada entre os anos de 2010 e 2019; e puder ser contactado via telefone, endereço eletrônico (e-mail) ou rede social. Foram excluídos da pesquisa os egressos que deixaram de responder mais de $20 \%$ do instrumento de coleta de dados.

Os egressos foram contactados e convidados a participar da pesquisa mediante contato telefônico e/ou endereço eletrônico, disponibilizados pela secretaria acadêmica. Para os egressos não localizados destas formas, foi realizada busca ativa em duas redes sociais (Facebook® e Instagram ${ }^{\circledR}$ ), bem como solicitou-se aos egressos já incluídos que disponibilizassem os contatos dos colegas de curso ainda não localizados.

A coleta de dados ocorreu de agosto de 2018 a agosto de 2020. Foi utilizado instrumento estruturado próprio, elaborado pela ferramenta Google forms ${ }^{\circledR}$, que compreendeu campos para preenchimento de dados: sociodemográficos (sexo, idade); profissionais relacionados ao primeiro emprego (área e local de atuação, tempo para conseguir o primeiro emprego, função exercida, regime de trabalho, faixa salarial, carga horária semanal). Os dados foram digitados e tabulados em planilhas eletrônicas do programa Microsoft Excel ${ }^{\circledR}$ 2016. Inicialmente procedeu-se a análise descritiva, através do cálculo de medidas de tendência central e dispersão, bem como frequências absolutas e relativas, com utilização do software Stata®, versão 12.

Realizou-se ainda análise de séries temporais, com a finalidade de apresentar a tendência do salário dos egressos em seu primeiro emprego, em comparação ao salário-mínimo nacional, ao longo dos anos de 2010 a 2019. Foi calculado o valor mínimo, médio e máximo de salário dos egressos em cada um dos anos pesquisados. Para a apresentação gráfica da análise, o alisamento da série foi realizado para a redução da variação aleatória com as médias móveis aritméticas de ordem 3, exceto nas extremidades da série que foi de ordem 2. A análise da tendência foi realizada com a regressão de Prais Winsten, que possui a correção de Durbin-Watson. Isto evitou a autocorrelação serial, considerando valor de $\mathrm{p}<0,05$ para identificação de tendência crescente (positiva) ou decrescente (negativa), caso contrário foi estacionária. A Average Annual Percent Change (AAPC), ou percentual de incremento anual, foi estimada com IC95\%, para facilitar a comparação entre as diferentes categorias analisadas, apresentando em porcentagem o incremento anual das taxas (Antunes \& Cardoso, 2020).

A pesquisa foi aprovada pelo Comitê de ética em pesquisa da instituição investigada, sob CAAE 87224318.3.0000.8156, e todos os preceitos da Resolução 466/2012 foram respeitados.

\section{Resultados}

Foram incluídos 143 participantes na pesquisa, de um total de 170 egressos. As idades variaram de 19 a 62 anos, com média de 30,1 $\pm 8,6$ anos. A maioria dos participantes eram mulheres $(93,7 \%, \mathrm{n}=134)$ e já se inseriram no mercado de trabalho $(91,6 \%, \mathrm{n}=131)$, sendo que 51,5\% $(\mathrm{n}=67)$ ingressaram na profissão no período de um a seis meses após a conclusão 
do curso, seguidos por 28,2\% ( $\mathrm{n}=37$ ) que tiveram sua inserção em período inferior a um mês do término do curso.

Em relação ao primeiro emprego, o maior quantitativo de egressos ingressou em instituições hospitalares $(73,3 \%, \mathrm{n}=$ 96), em serviços de alta complexidade - pronto socorro ou unidade de terapia intensiva $(19,8 \%, \mathrm{n}=26)$, na função de técnico em enfermagem $(73,3 \%, \mathrm{n}=96)$, com contratos regidos pela consolidação das leis do trabalho $(67,2 \%, \mathrm{n}=88)$ e com carga horária de 36 horas semanais $(43,5 \%, \mathrm{n}=57)$.

A maioria refere não possuir fonte de renda complementar ao emprego na área da enfermagem $(90,2 \%, \mathrm{n}=129)$. Dos que possuem renda complementar, quatro realizam cuidados domiciliares de idosos $(2,8 \%)$ e dois realizam plantões extras $(1,4 \%)$. Os demais têm renda complementar por atividades diversas à área da saúde.

No tocante a remuneração recebida no primeiro emprego na área da enfermagem, os participantes tiveram uma média de $\mathrm{R} \$ 1.702,92$, com mínimo de $\mathrm{R} \$ 790,00$ e máximo de $\mathrm{R}$ \$ 4.000,00. Do total de respondentes, 71 (49,7\%) recebiam salários abaixo da média encontrada, enquanto somente 39 (27,3\%) recebiam acima da média. O Gráfico 1 apresenta a remuneração recebida no primeiro emprego, bem como análise da série temporal em relação ao salário-mínimo nacional vigente em cada ano pesquisado.

Gráfico 1 - remuneração no primeiro emprego conforme ano de formação.

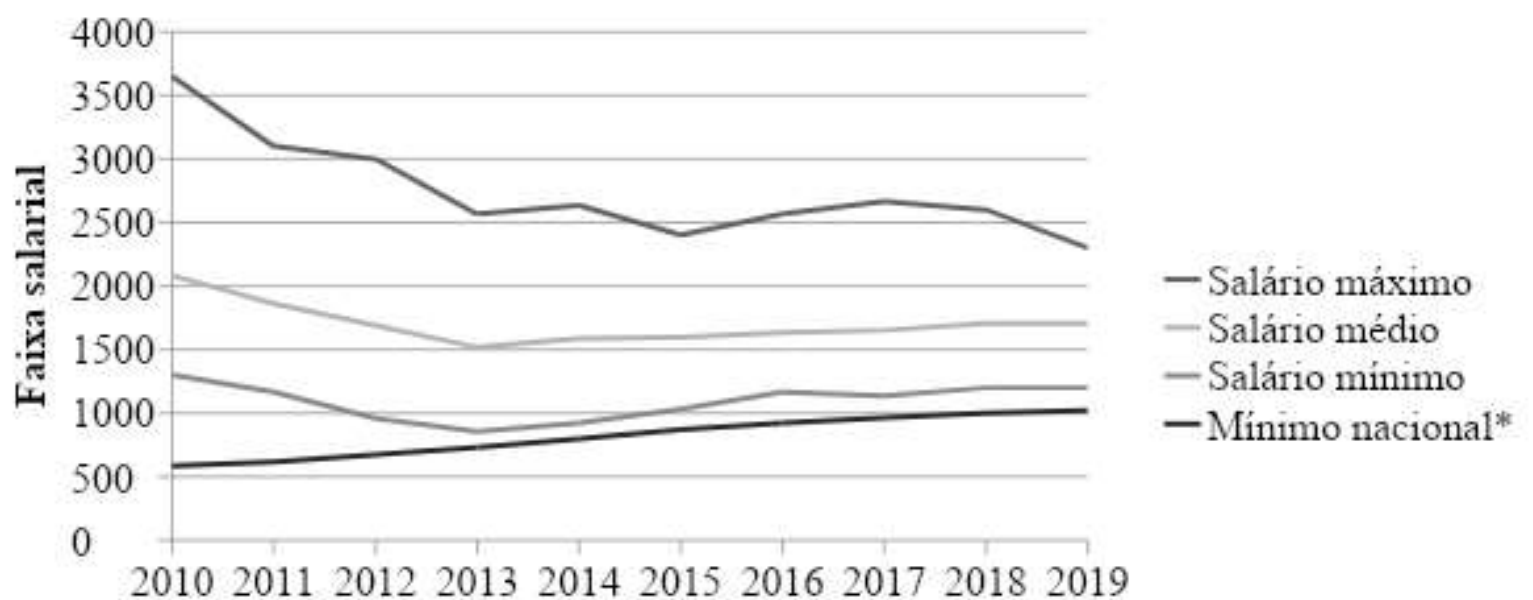

Ano de formação

\begin{tabular}{llllll}
\hline Categoria & AAPC $(\%)$ & AAPC $-(\%)$ & AAPC+ $(\%)$ & p-valor & Tendência \\
\hline Salário máximo & $-3,36$ & $-52,75$ & 6,90 & 0,09 & Estacionária \\
Salário médio & $-1,39$ & $-4,90$ & 2,25 & 0,399 & Estacionária \\
Salário mínimo & 0,52 & $-5,92$ & 7,41 & 0,861 & Estacionária \\
Salário-mínimo Nacional* & 7,46 & 5,72 & 9,23 & $<0,001$ & Crescente \\
\hline
\end{tabular}

Legenda: Average Annual Percent Change (AAPC) *valor do salário-mínimo no ano seguinte à conclusão do curso. Fonte: IPEA (2021).

Conforme observado no Gráfico 1 o salário-mínimo nacional teve uma tendência crescente de aumento nos dez anos pesquisados, enquanto o salário dos técnicos em enfermagem manteve tendência estacionária no mesmo período, tanto em relação aos valores máximo, médio, quanto mínimo recebidos. 


\section{Discussão}

A predominância feminina na profissão de enfermagem encontra-se bem assentada na literatura, o que corresponde atualmente a cerca de $80 \%$ da força de trabalho nesta área (Machado et al, 2015; Machado, 2017; Madalozo \& Artes, 2017), e que corrobora com esta pesquisa, cuja amostra é majoritariamente feminina, e relativamente jovem, com idade média de 30 anos. Estes resultados também se assemelham a outro estudo que pesquisou 40 alunos egressos de um curso técnico federal e encontrou população predominantemente feminina, com idade média semelhante (Gallo et al., 2015).

A presente pesquisa constatou que os técnicos em enfermagem ingressaram no mercado de trabalho em período inferior a seis meses após a conclusão de seu curso de formação e, destes, uma parcela significativa conseguiu esta inserção em menos de um mês após o término de sua formação. Em que pese o Brasil vivenciar um momento econômico desfavorável, com elevação da taxa de desemprego desde 2015, e com 14,8 milhões de brasileiros desempregados em 2021 (Instituto Brasileiro de Geografia e Estatística, 2021), pode-se afirmar que a empregabilidade na área da enfermagem apresenta-se em franca expansão, em especial nas capitais, pois a demanda por cuidado, nas diferentes áreas que a profissão contempla, requer cada vez mais profissionais. Neste cenário encontra-se a cidade de Curitiba, que se destaca como uma das capitais brasileiras com maior concentração de trabalhadores de enfermagem de nível médio atuando na área (Wermelinger et al, 2020), fato que sugere um desenvolvimento do setor de saúde na região pesquisada e consequente oportunidades profissionais.

Estudo nacional que avaliou a empregabilidade dos profissionais de enfermagem constatou que a força de trabalho tende a ser jovem e recém ingressada no mercado de trabalho, portanto ainda em processo de qualificação profissional (Machado, 2017), dado que vem ao encontro do apontado nesta pesquisa. Isto nos faz refletir sobre os motivos que levam a não permanência dos profissionais nesta área, dentre os quais a baixa remuneração poderia ser um dos fatores. No mais, autores apontam uma busca dos auxiliares e técnicos em enfermagem pelos cursos de graduação, uma vez que $78,1 \%$ deles referem cursar ou desejar cursar a graduação em enfermagem (Silva \& Machado, 2020), fato que pode estar ligado a busca por melhores salários.

Observou-se que a área hospitalar foi a que mais empregou os profissionais técnicos em enfermagem, geralmente colocados em áreas de alta complexidade, como pronto socorro e unidades de terapia intensiva. As áreas críticas, predominantes no emprego dos egressos, têm uma grande demanda de trabalhadores pois são um segmento importante de apoio e porta de entrada dos hospitais, detendo um expressivo contingente de trabalhadores. Salienta-se que as unidades hospitalares que atendem pacientes de alta complexidade demandam habilidades física e psicológica dos profissionais, o que, em virtude do desgaste que acarretam, desencadeiam alta rotatividade de trabalhadores, sendo então, locais onde geralmente há vagas disponíveis (Barbosa et al., 2011; Martins et al., 2019). No mais, o emprego em setores de alta complexidade pode acarretar pequeno aumento no salário, visto que, devido a maior exposição do trabalhador, o adicional de insalubridade previsto na Norma Regulamentadora número 15 costuma ser superior ao dos trabalhadores de outros setores de menor risco (Brasil, 1978).

A maioria dos pesquisados teve seu ingresso no mercado de trabalho pelo serviço privado, regido pela CLT. A maior incidência de profissionais empregados neste regime vai na contramão da realidade encontrada pelo Conselho Federal de Enfermagem, que identificou 57\% dos profissionais técnicos e auxiliares de enfermagem atuando no setor público (Machado, 2017), contudo tal achado pode ter relação com a diminuição de concursos públicos nos últimos anos, bem como com o fato de ser o primeiro emprego dos pesquisados.

A presente pesquisa apresenta a tendência dos salários recebidos pelos técnicos em enfermagem no primeiro emprego na região de Curitiba, estado do Paraná, apontando valores médios acima do salário-mínimo nacional vigente no país. Estudo realizado em um panorama nacional identificou que o salário dos técnicos em enfermagem seguiu o aumento do salário- 
mínimo nacional, contudo no período de 2013 a 2018 este aumento representou somente $0,7 \%$ em relação à média encontrada (Vieira et al, 2021), dado semelhante ao observado na presente pesquisa. Não obstante, apesar desta permanência superior ao salário-mínimo nacional, estes valores encontram-se aquém dos salários pretendidos por estes profissionais.

O Departamento Intersindical de Estatística e Estudos Socioeconômicos (DIEESE) realiza estudos nacionais que avaliam o poder de compra referente a um salário mínimo, bem como uma estimativa de qual deveria ser este valor, o que eles denominam 'salário mínimo necessário' (Departamento Intersindical de Estatística e Estudos Socioeconômicos, 2021), considerando como parâmetro o texto da Constituição Federal do Brasil, que estabelece que o salário mínimo deve atender às necessidades vitais básicas (moradia, alimentação, educação, saúde, lazer, vestuário, higiene, transporte e previdência social) do trabalhador e de sua família (Brasil, 1988). Segundo o DIEESE, o salário-mínimo necessário para atender a todas estas demandas deveriam ser de $\mathrm{R} \$ 2.194,36 \mathrm{em}$ janeiro de 2011 e de $\mathrm{R} \$ 4.347,61 \mathrm{em}$ janeiro de 2020 (Departamento Intersindical de Estatística e Estudos Socioeconômicos, 2021), anos estes tidos como base para o cálculo do mínimo nacional nesta pesquisa.

Estudo de base nacional cuja coleta de dados ocorreu com 35.914 profissionais de enfermagem de todas as categorias evidenciou uma proporção considerável de profissionais de enfermagem que recebem 'subsalários', ou seja, valores inferiores a R 1.000,00: 17,6\% da amostra, sendo que 1,8\% recebiam valor inferior ao salário-mínimo nacional na época (Machado, 2017). No mais, estudo que derivou deste primeiro aponta que os 'subsalários' são uma realidade em todos os setores, pois há $14,4 \%$ de profissionais que recebem menos de $\mathrm{R} \$ 1.000,00$ no serviço público, $22,1 \%$ no serviço privado e em proporção ainda maior nos serviços filantrópicos (23,7\%) (Silva \& Machado, 2020).

Sendo assim, fica evidente que o salário recebido pelos profissionais técnicos em enfermagem, em sua maioria, não atende ao mínimo necessário para o suprimento de suas necessidades básicas. Ou seja, uma profissão que se dedica ao cuidado da vida humana, desde seu nascimento até a sua finitude, não tem uma remuneração digna de suprir suas próprias necessidades vitais. No mais, a Constituição Federal estabelece que o salário deve sofrer reajustes frequentes, com vistas a preservar o poder aquisitivo dos indivíduos (Brasil, 1988), fato este que não foi observado junto aos pesquisados, em que a média salarial se manteve estacionário ao longo de dez anos.

Neste contexto, sugere-se que a predominância feminina, bem como o pouco tempo de formação profissional, podem ser fatores que contribuem para a baixa remuneração apresentada no primeiro emprego, identificada nesta pesquisa. Além disso, é necessário considerar as características socioeconômicas e culturais predominantes no perfil dos profissionais de enfermagem, desfavoráveis ao empoderamento para lutar por condições salariais condizentes com o exercício da profissão (Dias et al, 2019). Concorda-se com a visão de que para empresários, políticos, dirigentes de instituições de saúde, e até mesmo para população em geral, os profissionais de enfermagem são invisíveis, pois melhorias para seu trabalho e para sua saúde e de toda população não conseguem ser aprovadas (Silva \& Machado, 2020).

$\mathrm{O}$ trabalho na enfermagem sempre enfrentou dificuldades no que diz respeito à manutenção de salários dignos e condizentes com a preservação da qualidade de vida individual e/ou familiar (Schenone et al., 2018). O trabalho na enfermagem é desgastante, gera estresse e adoecimento, tanto de ordem psicológica quanto física (Silva \& Machado, 2020) e o fato de não ter condições financeiras para o lazer, para praticar atividade física, para uma boa alimentação, dentre outros fatores, compromete ainda mais a qualidade de vida destes profissionais.

De forma complementar, pondera-se que, na área da saúde, há a permanência de desequilíbrios salariais entre as profissões (Yeager \& Leider, 2017). Nas categorias profissionais de enfermagem isto vem desencadeando descontentamento profissional, estabelecendo a necessidade da intensificação de jornadas de trabalho: ou pelo duplo ou triplo emprego, ou pela realização de horas extras; o que pode inclusive desencadear exaustão emocional a estes trabalhadores, associados à 
diminuição dos períodos de lazer e convívio familiar (Schmidt et al, 2015; Santana et al., 2020). Em que pese neste estudo, os profissionais em sua maioria não possuíam fonte de renda complementar, contudo pesquisa de base nacional apontou que $11,2 \%$ dos profissionais de enfermagem complementam sua renda com outras atividades (Machado, 2017).

A enfermagem brasileira luta por uma remuneração digna e condizente com suas atribuições há anos. Registros jornalísticos baianos das décadas de 70 e 80 já destacavam o combate à desvalorização profissional e à sobrecarga de trabalho, bem como o esforço da classe por uma definição de jornada de trabalho e piso salarial (Laitano et al, 2019).

$\mathrm{Na}$ contemporaneidade diversos projetos de lei sobre o piso salarial foram propostos, no entanto não chegaram a ser votados pelo Congresso Nacional. Atualmente, encontra-se em tramitação no Senado Federal o Projeto de Lei 2.564/2020 (Brasil, 2020), que almeja a determinação de piso salarial para a categoria de enfermagem, na qual se propõe, para o técnico em enfermagem, uma remuneração de $\mathrm{R} \$ 5.120,00$. Este projeto passou por consulta pública, contando com mais de um milhão de manifestações favoráveis à sua aprovação.

Após reuniões em busca de apoio político para sua aprovação, um novo valor foi proposto. Na nova proposição, a remuneração do técnico em enfermagem corresponderá a $70 \%$ do piso salarial do enfermeiro, sugerido no valor de $\mathrm{R} \$$ 4.750,00. Entende-se que esta proposta é um caminho que pretende retificar a disparidade salarial, contudo a luta da categoria por reconhecimento e por remuneração dignas ainda persiste. De forma complementar, o projeto de lei indica a determinação de jornada de 30 horas semanais de trabalho para a categoria, sendo que nesta pesquisa a carga horária semanal relatada pelos profissionais foi de 36 horas.

Apontam-se que este tipo de pesquisa possui limitações como a autocorrelação serial na análise da série. Entretanto, foi utilizado o modelo de análise de Prais-Winsten, que minimiza ou elimina a autocorrelação da série. Uma das vantagens observadas é o seu potencial em suscitar importantes hipóteses acerca da estagnação salarial dos profissionais de enfermagem.

\section{Conclusão}

Em princípio, a remuneração dos técnicos em enfermagem encontra-se aquém do que anseia esta categoria profissional. A existência de um projeto de lei que determine não só o piso salarial da categoria, mas também sua jornada de trabalho, corrobora com a valorização destes trabalhadores; e sua implementação como política pública tende a fortalecer este coletivo de profissionais.

A baixa remuneração encontrada, pode estar relacionada com o perfil feminino da profissão e o pouco tempo de formação. Já a inserção profissional em locais de trabalho de alta complexidade pode viabilizar pequeno aumento salarial, em decorrência da insalubridade adicional.

A estagnação salarial dos técnicos em enfermagem evidenciada nas séries temporais, com tendência estacionária, pode representar um risco de "evasão" profissional destes trabalhadores para outras modalidades de trabalho, e/ou tornar desinteressante esta profissão aos que almejam ingressar na enfermagem de nível médio. Consequentemente, isto poderá sobrecarregar a saúde pública do país, tendo em vista que é uma classe profissional da saúde essencial para o funcionamento dos serviços de saúde, além de ser a mais numerosa do Brasil.

Sugere-se a realização de mais pesquisas que tenham como foco os profissionais de enfermagem de nível médio, de forma a contribuir para sua valorização, reconhecimento e melhoria de suas condições de trabalho e emprego.

\section{Referências}

Anderson, J. S. N. \& Schneider, S. (2014). Brazilian demographic transitionand the strategic role of youth. Espace Popul SocI [Internet], 2/3[24p]. https://doi.org/10.4000/eps.5774. 
Antunes, J. L. P. \& Cardoso, M. R. A. (2015). Uso da análise de séries temporais em estudos epidemiológicos. Epidemiol Serv Saúde [Internet],24(3):565-576. http://dx.doi.org/10.5123/S1679-49742015000300024.

Barbosa, T. L. A., Gomes, L. M. X., Reis, T. C. \& Leite, M. T. S. (2011). Expectativas e percepções dos estudantes do curso técnico em Enfermagem. Texto Contexto Enferm. 20(esp):45-51. https://www.scielo.br/j/tce/a/QkT73mpvwyV8cpkp9rwQCJp/?lang=pt\&format=pdf

Brasil (1978). Portaria 3.214, de 8 de junho de 1978. Norma regulamentadora número 15. Institui as atividades e operações insalubres. Diário Oficial da União 1978.

Brasil (1988). Constituição da República Federativa do Brasil, de 05 de outubro de 1988. Diário Oficial da União, 1988.

Brasil (2020). Projeto de Lei 2564/2020. Altera a Lei n ${ }^{\circ} 7.498$, de 25 de junho de 1986, para instituir o piso salarial nacional do Enfermeiro, do Técnico de Enfermagem, do Auxiliar de Enfermagem e da Parteira. Brasília (DF): Senado Federal; 2020.

Campoy, L. T., Ramos, A. C. V., Souza, L. L. L., Alves, L. S., Arcoverde, M. A. M., Berra, T. Z., Arroyo, L. H., Santos, D. T. \& Arcêncio, R. A. (2020). A distribuição espacial e a tendência temporal de recursos humanos para o Sistema Único de Saúde e para a Saúde Suplementar, Brasil, 2005 a 2016. Epidemiol Serv Saúde [Internet], 29(2). https://doi.org/10.5123/S1679-49742020000200020.

Cassiani, S. H. B. \& Lira Neto, J. C. G. (2018). Perspectivas da enfermagem e a campanha Nursing Now. Rev Bras Enferm 71(5):1-1. https://doi.org/10.1590/0034-7167.2018710501.

Departamento Intersindical de Estatística e Estudos Socioeconômicos (DIEESE) (2021). Pesquisa nacional da cesta básica de alimentos: salário-mínimo nominal e necessário. https://www.dieese.org.br/analisecestabasica/salarioMinimo.html

Dias, M. O., Souza, N. V. D. O., Penna, L. H. G. \& Gallasch, C. H. (2019). Percepção das lideranças de enfermagem sobre a luta contra a precarização das condições de trabalho. Rev Esc Enferm USP 53:e03492. https://doi.org/10.1590/S1980-220X2018025503492

Gallo, A. M., Natal, A. A. E., Silva, D. O., Salvagioni, D. A. J. \& Araujo, J. P. (2015). O egresso técnico em enfermagem e sua inserção no mundo do trabalho. Anais Eletrônico IX EPCC - Encontro Internacional de Produção Científica UniCesumar, 9:4-8. http://www.cesumar.br/prppge/pesquisa/epcc2015/anais/juliane_pagliari_araujo_2.pdf

Giordano, V. D. (2020). Contrastando organizações de serviços de saúde públicas e privadas: a experiência dos gestores [dissertação]. Lisboa (Portugal): Instituto Universitário de Lisboa; 2020. http://hdl.handle.net/10071/21922

Instituto Brasileiro de Geografia e Estatística - IBGE (2021). Pesquisa nacional por amostra de domicílios contínua. IBGE, 2021. https://www.ibge.gov.br/estatisticas/sociais/trabalho/9173-pesquisa-nacional-por-amostra-de-domicilios-continua-trimestral.html?=\&t=serieshistoricas\&utm_source=landing\&utm_medium=explica\&utm_campaign=desemprego

Instituto de Pesquisa Econômica Aplicada - $\quad$ IPEA (2021). Salário mínimo vigente. IPEA, 2021. http://www.ipeadata.gov.br/exibeserie.aspx?stub=1\&serid1739471028=1739471028

Laitano, A. D. C., Silva, G. T. R., Almeida, D. B., Santos, V. P. F. A., Brandão, M. F., Carvalho, A. G., Peres, M. A. A. \& Santana, N (2019). Precarização do trabalho da enfermeira: militância profissional sob a ótica da imprensa. Acta Paul Enferm 32(3):305-311. http://dx.doi.org/10.1590/1982- 0194201900042

Machado, M. H., Aguiar Filho, W., Lacerda, W. F., Oliveira, E., Lemos, W., Wermelinger, M., Vieira, M., Santos, M. R., Souza Junior, P. B., Justino, E. \& Barbosa, C (2015). Características gerais da enfermagem: o perfil sociodemográfico. Enferm Foco 6(1/4):11-17. https://doi.org/10.21675/2357707X.2016.v7.nESP.686.

Machado, M. H. (Coord) (2017). Perfil da enfermagem no Brasil: relatório final: Brasil. NERHUS - DAPS - ENSP/Fiocruz, 2017. http://www.cofen.gov.br/perfilenfermagem/pdfs/relatoriofinal.pdf

Madalozzo, R. \& Artes, R. Escolhas profissionais e impactos no diferencial salarial entre homens e mulheres. Cad Pesqui 47(163):202-221. http://dx.doi.org/10.1590/198053143666.

Martins, M. S., Matos, E. \& Salum, N. C. (2019). Rotatividade dos trabalhadores de enfermagem em uma unidade de emergência adulto. Texto Contexto Enferm 28:e20160069. https://dx.doi.org/10.1590/1980-265X-TCE-2016-0069.

Santana, L. L., Sarquis, L. M. M. \& Miranda, F. M. D. (2020). Riscos psicossociais e a saúde dos trabalhadores de saúde: reflexões sobre a reforma trabalhista brasileira. Rev Bras Enferm 73(sp1):e20190092. http://dx.doi.org/10.1590/0034-7167-2019-0092.

Schenone, D., Rasero, L. \& Cavaliere, B. (2018). Fee payment system for nursing performance: an operational proposal through the use of I.C.A. (Indexes of Complexity of Assistance) methodology. Acta Biomed 89(sp 6):87-96. https://doi.org/10.23750/abm.v89i6-S.7451.

Schmidt, D. R. C., Paladini, M., Biato, C., Pais, J. D. \& Oliveira, A. R. (2013). Qualidade de vida no trabalho e burnout em trabalhadores de enfermagem de unidade de terapia intensiva. Rev Bras Enferm [Internet] 66(1):13-17. https://doi.org/10.1590/S0034-71672013000100002.

Silva, M. C. N. (2019). O Nursing Now desembarca no Brasil para evidenciar a força e a capacidade da Enfermagem. Enferm foco 10(1):01-01. http://revista.cofen.gov.br/index.php/enfermagem/article/view/2322/494

Silva, M. C. N. \& Machado, M. H. (2020). Sistema de saúde e trabalho: desafios para a enfermagem no Brasil. Ciênc Saúde Colet 25(1):7-13. https://doi.org/10.1590/1413-81232020251.27572019.

Vieira, L. J. E. S., Silva, R. M., Garcia Filho, C., Bezerra, I. C., Ferreira Junior, A. R. \& Guimarães, J. M. X. (2021). Trabalho em enfermagem: análise da tendência dos salários no Brasil. Res Soc Develop 10(3):e54210313569. https://doi.org/10.33448/rsd-v10i3.13569. 
Research, Society and Development, v. 10, n. 16, e346101623840, 2021

(CC BY 4.0) | ISSN 2525-3409 | DOI: http://dx.doi.org/10.33448/rsd-v10i16.23840

Wermelinger, M. C. M. W., Boanafina, A., Machado, M. H., Vieira, M., Ximenes Neto, F. R. G. \& Lacerda, W. F. (2020). A formação do técnico em enfermagem: perfil de qualificação. Ciênc Saúde Colet 25(1):67-78. https://doi.org/10.1590/1413-81232020251.27652019.

Yeager, V. A. \& Leider, J. P. (2019). The role of salary in recruiting employees in state and local governmental public health: PH WINS 2017. Am J Public Health 109(5):683-685. https://doi.org/10.2105/AJPH.2019.305008. 